\title{
Central-Type Fusion-based Methods for Degraded Image Understanding ${ }^{*}$
}

\author{
Zhang Cui ${ }^{1}$, Zhang Xiujun ${ }^{2}$, Zhao Shengying ${ }^{1}$, Shang Lina ${ }^{1}$, and Xiong Kai ${ }^{1}$ \\ ${ }^{1}$ School of Information \& Electrical Engineering, Zhejiang University City College, Zhejiang, China \\ ${ }^{2}$ School of Applied Engineering School, Zhejiang Vocational College of Commerce, Zhejiang, China \\ zhangcui523@126.com, seesky88@126.com
}

\begin{abstract}
Information fusion in target recognition is widely used, and application of information fusion in image field is developing continuously. When the degraded image is acquired, we often use the clearness algorithm to enhance image quality, Then the image of the target is identified, due to the lack of clarity of the image itself, the recognition accuracy is not high. Using a fusion rule of Dempster-Shafer evidence theory, the recognition algorithm of different posterior credibility is fusion, in order to get better recognition effect. This paper provides a fusion method for target recognition, the target exists in the degraded image. the fusion method is based on Central-Type algorithm of DS evidence theory. The results show that the method can improve the probability of correct recognition, reduce the error probability.

Index Terms - Degraded image, Dempster-Shafer evidence theory, Central-Type Fusion, Target recogniton
\end{abstract}

\section{Introduction}

Target recognition is an algorithm, that shows what is the target[1]. The attribute data of target come from different sensor. There is a set including the attribute data of the target. Target recognition algorithm is divided into three types: physical model, The method based on knowledge, Parameter classification technology[2]. There are many ways of data fusion, in which Bayes and Dempster-Shafer method is the most active in research and use, attracted special attention[3].

Images obtained in rain and fog weather is often degraded image, but Clear image is more able to meet the needs of detection and recognition. In recent years, many domestic and foreign scholars study the enhancement algorithm for degraded image, the contents of the research include the image clearness algorithm, contrast enhancement method, degradation image restoration method[4]. No matter what kind of algorithm, there have the advantages and disadvantages.

Target recognition is advanced application of image detection technology, There are a variety of identification algorithms, including the algorithm based on neural network and the algorithm based on feature extraction, etc[5]. No matter what kind of target recognition algorithm, there have the advantages and disadvantages.

For the enhanced image of the target, when you use different recognition algorithms, the accuracy of identification is different, because of the diversity of enhancement and recognition method[6]. In this paper, the DS evidence theory fusion algorithm and enhancement and recognition algorithm are combined, the reliability of distribution is integrated, so as to improve the accuracy of recognition.

\section{The Basic Theory of Dempster-Shafer Evidence Theory}

Assuming that $\Omega$ is the sample space, Proposition in the field can use a subset of the $\Omega$.

Definition 1: Suppose that the function M: $2^{\Omega} \rightarrow[0,1]$, and require $M(\Phi)=0, \sum_{A \subseteq \Omega} M(A)=1$

Then $\mathrm{M}$ is the probability distribution function of the $2^{\Omega}$, $\mathrm{M}$ (A), referred to as A basic probability, This means that A precise trust.

Definition 2: The proposition trust function Bel: $2^{\Omega} \rightarrow$ $[0,1]$, and $\operatorname{Bel}(\mathrm{A})=\sum_{B \leq A} M(B)$, for all $\mathrm{A} \subseteq \Omega$

The Bel function is also called the lower limit function, It means that full trust to A, It is easy to get, using the definition of probability distribution function $\operatorname{Bel}(\Phi)=\mathrm{M}(\Phi)=0$, $\operatorname{Bel}(\Omega)=\sum_{B \subseteq \Omega} M(B)$

Definition 3: Likelihood function P1: $2^{\Omega} \rightarrow$ [0,1], and $\mathrm{P} 1 \mid(\mathrm{A})=1-\mathrm{Bel}(-\mathrm{A})$, for all $\mathrm{A} \subseteq \Omega$

Definition 4: Suppose M1 and M2 are two probability distribution function in the $\Omega$, then orthogonal and $\mathrm{M}=\mathrm{M} 1+$ M2 defined as

$$
\begin{aligned}
& \mathrm{M}(\Phi)=0, \mathrm{M}(\mathrm{A})=\mathrm{c}^{-1} \sum_{x \cap y=A} M_{1}(x) M_{2}(x), \mathrm{A} \neq \Phi \\
& \text { Among them, } \mathrm{c}=1-\sum_{\cap A i=\Phi} \prod_{1 \leq i \leq n} M_{i}\left(A_{i}\right)
\end{aligned}
$$

\section{Fusion Method Based on Dempster-Shafer Evidence Theory}

A. the fusion probability assignment for individual sensor, in multiple measuring cycles

Assume that sensors in each measurement cycle of the a posteriori probability assignment (These posteriori probability assignment is obtained through continuous target situation and fixed a priori probability assignment) is, M1(Ai), M2(Ai),..... Mn (Ai), i = 1, 2, ..... k

Wherein, $\mathrm{Mj}$ ( $\mathrm{Ai}$ ) is the reliability of distribution of propositional $\mathrm{Ai}$ value in the $\mathrm{j}$ cycle, in addition, ui is the probability assignment of the proposition of the "unknown"

\footnotetext{
* This work is partially supported by Zhejiang Provincial Department of education research project Y201223096, supported by Zhejiang Provincial Natural Science Foundation of China LY12F01017 and Y1110632, supported by Teacher Scientific Research Fund (J-12006).
} 
value in the $n$-th cycle. Posteriori confidence measure of the $k$ th proposition were obtained through the accumulation of the sensor $\mathrm{n}$ measurement cycle test. Posteriori confidence measure after fusion is,

$$
\begin{aligned}
& M(A i)=c^{-1} \sum_{\cap A_{j}=i} \prod_{1 \leq \mathrm{s} \leq \mathrm{n}} M_{1 j}(A i), \mathrm{i}=1,2, \ldots \ldots \mathrm{k} \\
& \text { Among them, } \quad c=1-\sum_{\cap A i=\Phi} \prod_{1 \leq \mathrm{s} \leq \mathrm{n}} M_{s}(A i)
\end{aligned}
$$

Special, the probability assignment of the proposition of the "unknown" value is, $\mathrm{u}=\mathrm{u} 1 \mathrm{u} 2 \ldots . \mathrm{un} / \mathrm{c}$

For addition to the "unknown" proposition, various propositions are independent of each other circumstances, Formula (2) can be transformed into

$$
\begin{aligned}
\mathrm{M}(\mathrm{Ai}) & =\{\mathrm{M} 1(\mathrm{Ai})[\mathrm{M} 2(\mathrm{Ai})+\mathrm{u} 2][\mathrm{M} 3(\mathrm{Ai})+\mathrm{u} 3] \ldots[\mathrm{Mn}(\mathrm{Ai})+\mathrm{un}] \\
& +\mathrm{u} 1 \mathrm{M} 2(\mathrm{Ai})[\mathrm{M} 3(\mathrm{Ai})+\mathrm{u} 3][\mathrm{M} 4(\mathrm{Ai})+\mathrm{u} 4] \ldots[\mathrm{Mn}(\mathrm{Ai})+\mathrm{un}] \\
& +\mathrm{u} 1 \mathrm{u} 2 \mathrm{M} 3(\mathrm{Ai})[\mathrm{M} 4(\mathrm{Ai})+\mathrm{u} 4][\mathrm{M} 5(\mathrm{Ai})+\mathrm{u} 5] \ldots[\mathrm{Mn}(\mathrm{Ai})+\mathrm{un}] \\
& +\ldots+\mathrm{u} 1 \mathrm{u} 2 \ldots \mathrm{un}-2 \mathrm{Mn}-1(\mathrm{Ai})[\mathrm{Mn}(\mathrm{Ai})+\mathrm{un}] \\
& +\mathrm{u} 1 \mathrm{u} 2 \ldots \mathrm{un}-1 \mathrm{Mn}(\mathrm{Ai}) \\
& =c^{-1} \sum_{j=1}^{n}\left\{M_{j}(\mathrm{Ai})\left[\prod_{s=0}^{j-1}\right]\left[\prod_{s=j+1}^{n} \mathrm{M}_{\mathrm{s}}(\mathrm{Ai})+\mathrm{u}_{\mathrm{s}}\right]\right\}
\end{aligned}
$$

Among them, $\mathrm{u} 0=0$, and

$$
c=\sum_{j=1}^{n}\left\{M_{j}(A i)\left[\prod_{s=0}^{j-1}\right]\left[\prod_{s=j+1}^{n} \mathrm{M}_{\mathrm{s}}(\mathrm{Ai})+\mathrm{u}_{\mathrm{s}}\right]\right\}+\prod_{s=1}^{n}
$$

B. the fusion probability assignment for multiple sensors, in more than one measurement cycle

Suppose there are $\mathrm{m}$ sensors. The a posteriori Credits allocation of Each sensor in each measurement cycle is

$$
\begin{aligned}
& M_{s j}\left(A_{i}\right) \quad \mathrm{i}=1,2, \ldots, \mathrm{k}, \mathrm{j}=1,2, \ldots, \mathrm{n}, \mathrm{s}=1,2, \ldots, \mathrm{m} \\
& u_{s j}=M_{s j}(\Omega) \quad \mathrm{j}=1,2, \ldots, \mathrm{n}, \mathrm{s}=1,2, \ldots, \mathrm{m}
\end{aligned}
$$

$M_{s j}\left(A_{i}\right)$ is the posterior probability assignment for proposition $\mathrm{Ai}$, s sensor in the $\mathrm{j}$ measurement cycles of $\mathrm{A}$, in this case it is for the s-th sensors in the $\mathrm{j}$-th measurement cycle, usj is the probability assignment of the proposition of the "unknown" value. The following will be discussed that the fusion probability assignment for multiple sensors, in more than one measurement cycle. The algorithm includes two methods, one is a Central- Type computing, and the other is a distributed computing. The paper uses the method of CentralType Computing.

The main idea Central-Type computing is that, in the DS evidence theory, the first, computing the fusion probability assignment for individual sensor, in multiple measuring cycles, then computing the fusion of the sum of all sensor. In the image recognition algorithm, each sensor is equivalent to every kind of recognition, such as the recognition algorithm based on neural network and feature based recognition algorithm. Different period is equivalent to different enhancement algorithms, such as image sharpening algorithm, contrast enhancement method, degraded image restoration method, etc. Three kinds of recognition algorithm and three enhancement algorithm for example, center computing model as shown in figure 1 .

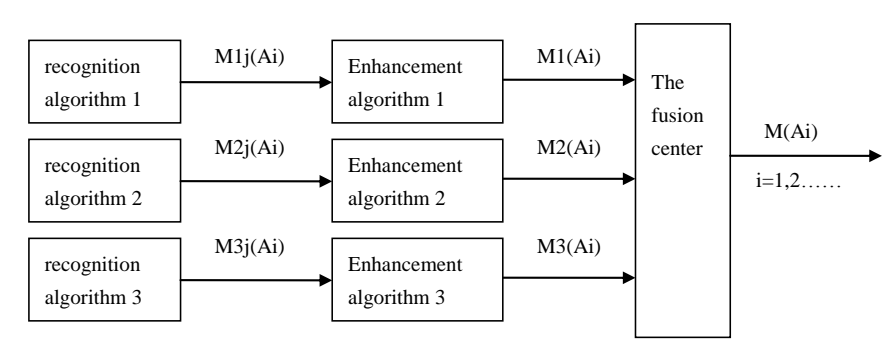

Fig. 1 Central-Type computing

\section{Simulation}

A. the fusion probability assignment for individual sensor, in multiple measuring cycles

We use MATLAB language to complete the simulation. MATLAB has very high efficiency in image processing.

Here are three images, each image have been enhanced through different enhancement algorithm. It will recognize the specified target in which each image, using the recognition algorithm. Assuming the identification algorithm for identification of different enhancement algorithm for a priori Credits allocation data is :

M1(\{ Torpedo $\},\{$ Shoal of fish $\},\{$ Unknown object $\})=(0.7,0.2,0.1)$

M2(\{ Torpedo $\},\{$ Shoal of fish $\},\{$ Unknown object $\})=(0.6,0.2,0.2)$

Table $\mathrm{I}$ is comparison of the fusion probability assignment, it fusing the probability assignment of the single recognition algorithm for the object in image, and the image is enhanced thought different enhancements.

TABLE I The fusion for individual sensor, in multiple measuring cycles

\begin{tabular}{|c|c|c|c|}
\hline & $\begin{array}{c}\text { Priori } \\
\text { credibility 1 }\end{array}$ & $\begin{array}{c}\text { Priori } \\
\text { credibility 2 }\end{array}$ & $\begin{array}{c}\text { Priori } \\
\text { credibility 3 }\end{array}$ \\
\hline $\mathrm{M}$ (Torpedo) & 0.7 & 0.6 & 0.8378 \\
\hline $\mathrm{M}$ (Shoal of fish) & 0.2 & 0.2 & 0.1351 \\
\hline $\mathrm{M}$ (Unknown object) & 0.1 & 0.2 & 0.0270 \\
\hline
\end{tabular}

It can be seen that the possibility of torpedo target, according to the fusion probability assignment. It Is more accurate than the reliability distribution of intuitive.

B. the Central-Type fusion probability assignment for multiple sensors, in more than one measurement cycle

Here are two images, the first image uses the first enhanced algorithm, the second image uses the second enhanced algorithm. It will recognize the specified target in the first image, using the three different recognition algorithm, then the second image too. Assuming the identification algorithm for identification of different enhancement algorithm for a priori Credits allocation data is : 
recognition algorithm 1:

M11( $\{$ Torpedo $\},\{$ Shoal of fish $\},\{$ Unknown object $\})$

$=(0.7,0.2,0.1)$

M12( $\{$ Torpedo $\},\{$ Shoal of fish $\},\{$ Unknown object $\})$

$=(0.6,0.2,0.2)$

recognition algorithm 2 :

M21( $\{$ Torpedo \}, \{ Shoal of fish \}, \{ Submarine \},

$\{$ Unknown object $\})=(0.5,0.2,0.1,0.2)$

M22(\{ Torpedo \}, \{ Shoal of fish \}, \{ Submarine \},

$\{$ Unknown object $\})=(0.6,0.2,0.1,0.1)$

recognition algorithm 3:

M31 $(\{$ Torpedo $\},\{$ Unknown object $\})=(0.6,0.4)$

M32 $(\{$ Torpedo $\},\{$ Unknown object $\})=(0.4,0.6)$

Table II is comparison of the fusion probability assignment, it fusing the probability assignment of the three different recognition algorithm for the object in image, and the image is enhanced thought the two different enhancements.

TABLE II The fusion for multiple sensor, in multiple measuring cycles

\begin{tabular}{|c|c|c|c|c|c|}
\hline \multicolumn{2}{|c|}{} & $\mathrm{M}$ (Torpedo) & $\begin{array}{c}\mathrm{M} \text { (Shoal of } \\
\text { fish) }\end{array}$ & $\begin{array}{c}\mathrm{M} \text { (Sub } \\
\text { marine) }\end{array}$ & $\begin{array}{c}\mathrm{M} \text { (Unknown } \\
\text { object) }\end{array}$ \\
\hline $\begin{array}{c}\text { recognition } \\
\text { algorithm 1 }\end{array}$ & $\begin{array}{c}\text { Image } \\
\text { enhancement 1 }\end{array}$ & 0.7 & 0.2 & 0 & 0.1 \\
\cline { 2 - 6 } & $\begin{array}{c}\text { Image } \\
\text { enhancement 2 }\end{array}$ & 0.6 & 0.2 & 0 & 0.2 \\
\hline $\begin{array}{c}\text { The two fusion for recognition } \\
\text { algorithm 1 }\end{array}$ & 0.8378 & 0.1351 & 0 & 0.0270 \\
\hline $\begin{array}{c}\text { recognition } \\
\text { algorithm 2 }\end{array}$ & $\begin{array}{c}\text { Image } \\
\text { enhancement 1 }\end{array}$ & 0.5 & 0.2 & 0.1 & 0.2 \\
\cline { 2 - 6 } & $\begin{array}{c}\text { Image } \\
\text { enhancement 2 }\end{array}$ & 0.6 & 0.2 & 0.1 & 0.1 \\
\hline $\begin{array}{c}\text { The two fusion for recognition } \\
\text { algorithm 2 }\end{array}$ & 0.7460 & 0.1587 & 0.0635 & 0.0270 \\
\hline $\begin{array}{c}\text { recognition } \\
\text { algorithm 3 }\end{array}$ & $\begin{array}{c}\text { Image } \\
\text { enhancement 1 }\end{array}$ & 0.6 & 0 & 0 & 0.4 \\
\cline { 2 - 6 } & $\begin{array}{c}\text { Image } \\
\text { enhancement 2 }\end{array}$ & 0.4 & 0 & 0 & 0.6 \\
\hline $\begin{array}{c}\text { The two fusion for recognition } \\
\text { algorithm3 }\end{array}$ & 0.7600 & 0 & 0 & 0.2400 \\
\hline \begin{tabular}{c} 
The three fusion \\
\hline
\end{tabular} & 0.96732 & 0.0131 & 0.00058295 & 0.00024815 \\
\hline
\end{tabular}

It can be seen from the table that credibility of recognition algorithm 1 ( 2 or 3 ) to enhance image recognition algorithm is higher than of distribution ratio 1 ( 2 or 3 ) of any two consecutive recognition. The probability assignment of the fusion for the three different recognition algorithm is higher than the probability assignment of anyone recognition algorithm. In addition, it can also be seen that The target is most likely to $M$ (torpedo) relative to $M$ (Shoal of fish), $\mathrm{M}$ (Submarine) and M(Unknown object). And it has the smallest possibility that the target is unidentified objects.

In the image recognition, data fusion algorithm can also be used. We can fuse posteriori probability assignment values. These values are obtained by different recognition algorithms. The fusion method based on DS evidence theory. It can get better recognition effect. The extracted characteristics by single recognition algorithm can not be a complete description of a target, Because of its recognition algorithm defects. So, you can not make full use of goal-related information. It affects the validity and reliability of the feature set. In this case, the target recognition system performance is not satisfactory. The method in this paper can be more complete description of the target, Because we have many different identification image and several different enhancements, and independent, complementary to the feature vector is extracted, then we have adopted a comprehensive treatment technology. Simulations show that this method is beneficial to improve the probability of correct identification, to reduce the error probability.

\section{V.Acknowledgment}

This work is supported by Zhejiang Provincial Department of education research project Y201223096, supported by Zhejiang Provincial Natural Science Foundation of China LY12F01017 and Y1110632, supported by Teacher Scientific Research Fund (J-12006).

\section{References}

[1] Shi Wenjun, Wang Xiaoping, and Wang dengwei, "Alongshore Ship Detection Based on D-S Evidence Theory," Electronics Optics \& Control, vol. 16, no. 11,pp.87-91, November 2009

[2] Zhou Fang, and Wang Pengbo, "Multi-source knowledge fusion algorithm," Journal of Beijing University of Aeronautics and Astronautics, vol.39, no 1, pp. 109-114, January 2013

[3] Li Yanmei, Rao Yunbo, Chen Leiting, and Zhou Jun, "Color image enhancement method using genetic algorithm," Journal of Computational Information Systems, vol. 8, no. 19, pp. 8065-8073, October 2012

[4] Hu Hongqing, and Ni Guoqiang, "The improved algorithm for the defect of the Retinex image enhancement," 2010 International Conference on Anti-Counterfeiting, Security and Identification, pp. 257-260, 2010.

[5] Zhang Yunfeng, Zhang Caiming, Chi Jing, and Zhang Rui," An algorithm for enlarged image enhancement," ICIC Express Letters, vol. 3, no. 3, pp. 669-674, September 2009.

[6] Blasch. Erik, and Huang. Sam," Multilevel feature-based fuzzy fusion for target recognition," The International Society for Optical Engineering, vol. 4051 , pp. $279-288,2000$ 